\title{
Compressive cryotherapy versus cryotherapy alone in patients undergoing knee surgery: a meta-analysis
}

Mingzhi Song ${ }^{1,2+}$, Xiaohong Sun ${ }^{1,3+}$, Xiliang Tian ${ }^{1}$, Xianbin Zhang ${ }^{4}$, Tieying Shi ${ }^{3}$, Ran Sun ${ }^{3,5^{*}}$ and Wei Dai ${ }^{3,5^{*}}$

\begin{abstract}
Aim: This study aims to conduct a meta-analysis to identify and compare the effectiveness of compressive cryotherapy and cryotherapy alone for patients undergoing knee surgery.

Background: Postoperative management is an important guarantee for the success of surgery. Cryotherapy and compression are two common nursing techniques after knee surgery, and are considered to be effective for postoperative clinical symptoms such as local pain and swelling. However, no previous meta-analyses have compared the effectiveness of compressive cryotherapy and cryotherapy alone in patients undergoing knee surgery.
\end{abstract}

Design: A meta-analysis of randomized controlled trials (RCTs).

Methods: We conducted a search in MEDLINE (via Pubmed, 1990-2014), EMBASE (via Elsevier, 1990-2014), Cochrane Central Register of Controlled Trials (The Cochrane Library, 1990-2014), CINAHL (1990-2014) and China National Knowledge Infrastructure (1990-2014) databases for RCTs published in English and Chinese. The primary outcome measure of interest was visual analog scale and girth measure. Finally, a meta-analysis was carried out using RevMan 5.3.

Results: Among the 593 RCTs, 10 RCTs were selected and included into this study. These studies included 522 patients who underwent knee surgery. Patients who underwent compressive cryotherapy tended to have less pain than patients who underwent cryotherapy alone at POD2 and POD3, while compressive cryotherapy had a strong tendency towards less swelling over cryotherapy alone at POD1 and POD2. However, there was no significant difference between compressive cryotherapy and cryotherapy alone at the intermediate stage of rehabilitation after knee surgery. All adverse reactions were recorded in all included RCTs.

Conclusion: Current evidence suggests that compressive cryotherapy is beneficial to patients undergoing knee surgery at the early rehabilitation stage. At the last stage, the effectiveness of compressive cryotherapy and cryotherapy alone were found to be similar.

Keywords: Cryotherapy, Meta-analysis, Nursing, Surgery, Total knee arthroplasty, Anterior cruciate ligament reconstruction, Arthroscopy, Postoperative care, Postoperative complications, Pain, Edema, Pain relief

*Correspondence: sunran_dmu@126.com; daiwei_dmu@126.com

${ }^{\dagger}$ Mingzhi Song and Xiaohong Sun are co-first authors

${ }^{5}$ Operation Room, The First Affiliated Hospital of Dalian Medical

University, 222 Zhong Shan Road, Dalian 116011, Liaoning, People's

Republic of China

Full list of author information is available at the end of the article 


\section{Background}

Total knee arthroplasty (TKA), knee arthroplasty, anterior cruciate ligament (ACL) reconstruction and arthroscopic therapy have become the most common knee surgical methods. Numerous patients suffering from knee diseases have achieved satisfactory curative effects through these methods. However, severe clinical symptoms including local pain, swelling and reduced knee range of motion have frequently occurred during the postoperative period (van Grinsven et al. 2010; Yabroudi and Irrgang 2013). They were generally treated as main factors that delay functional recovery time. Therefore, effective postoperative management appears to be particularly important for the operative success that surgeons have contributed.

Cryotherapy or cold treatment is the traditional treatment, which is affordable, easy to perform and widely applied for acute musculoskeletal injuries. Cold has extensive roles in tissue injury recovery, which mainly include reducing cellular metabolism, delaying nerve conduction, inhibiting edema expansion and alleviating pain (Nadler et al. 2004; Warren et al. 2004; Cohn et al. 1989). To some extent, surgery was been regarded as an acute injury. Therefore, cryotherapy has been gradually applied in postoperative patients. In addition to relieving pain and edema, cryotherapy is also helpful in promoting healing; enabling patients to return to their regular activities. Several researchers have achieved these exact results, which were obtained through comparative studies that evaluated the effectiveness of cryotherapy in patients after knee surgeries (Cohn et al. 1989; Lessard et al. 1997). Evidence-based medicine studies have indicated that patients undergoing TKA and arthroscopic ACL reconstruction have benefited from postoperative cryotherapy (Ni et al. 2015; Adie et al. 2010; Martimbianco et al. 2014). Since nurses have begun to be involved in decision-making, cryotherapy has become an important postoperative management. Through thousands of years of development and evolvement, cryotherapy could now be applied through different methods such as cold dressing, cold packs, crushed ice bags, cooling pads and cold compression devices (CCD). Due to the facilitation of the operation process, more nurses have continued to select CCD to perform cryotherapy. In fact, compared with traditional cryotherapy, CCD simultaneously brings compression into postoperative management. Several clinical studies on the effectiveness of compression have been performed without inconclusive results, and compression remains to be seen as a common postoperative intervention (Charalambides et al. 2005; Smith et al. 2002; Andersen et al. 2008; Pinsornsak and Chumchuen 2013; Cheung et al. 2014; Munk et al. 2013). To date, compressive cryotherapy (CC) has been shown to significantly reduce postoperative pain scores after TKA, ACL reconstruction and wrist arthroscopy (Meyer-Marcotty et al. 2011; Markert 2011; Schröder and Pässler 1994). Although cryotherapy has exhibited a clear effect in promoting recovery for postoperative patients, few studies have compared $\mathrm{CC}$ and cryotherapy alone (CA). For guidance in postoperative nursing, this kind of comparison has become more significant. The viewpoint on this comparison is very inconsistent in academia at present. The study of Kraeutler et al. indicated that CC by CCD did not reduce postoperative pain in patients undergoing shoulder arthroscopy (Kraeutler et al. 2015). However, the opposite view for $\mathrm{CC}$ has also attracted the people's attention (Schröder and Pässler 1994). Fortunately, studies that have compared CC and CA in patients after knee surgery are relatively enough to attain a conclusion. Since randomized controlled trials (RCTs) have been published over the past 24 years, we decided to critically appraise and synthesize existing evidences obtained when effectiveness was compared between compressive cryotherapy and cryotherapy alone following knee surgery. This review would help explore the impact of compression on cryotherapy, assist in clinical and nursing decisions for selecting the optimum cryotherapeutic method, and study the gaps in this area.

\section{Aim and methods}

This study conducted a meta-analysis to identify and compare the effectiveness of $\mathrm{CC}$ and $\mathrm{CA}$ in patients undergoing knee surgery. For this study, the review process of the Cochrane Collaboration was adopted, including the identification of a priori inclusion/exclusion criteria (Higgins and Green 2011). Furthermore, the review included the development of a structured clinical question linked to the comprehensive and detailed search of literature using appropriate databases and a priori inclusion and exclusion criteria, the systematic extraction and recording of study characteristics, methods, findings and methodological qualities, and the synthesis of comparable studies. Two independent reviewers were involved in each stage, and their extractions and appraisals were cross-referenced to ensure accuracy.

\section{Eligibility criteria}

Randomized controlled trials were selected for this study. Skeletally mature patients (18 years old) were submitted to primary TKA, knee arthroplasty, ACL reconstruction and arthroscopic surgery. Studies that included patients with bilateral and secondary surgery were excluded. Any type of compressive cryotherapy around the knee (e.g. CCD and home-made equipment) compared to cryotherapy alone around the knee (e.g. cold dressing, cold packs, cooling pads, crushed ice bags and CCD) were 
interventions that needed to be studied. CCD is a compressive cooling system [e.g. Cryocuff system (Aircast Cryocuff, Inc., Summit, New Jersey, USA), Game ready (CoolSystems, Inc., Alameda, California, USA) coolsystems and Ever-cryo system (Cryo-Push Medical Technology Co., Ltd., Chengdu, Sichuan, China)] that can supply a controlled cryogenic circulation and generate focal compression to the knee.

\section{Outcome measures}

(1) Pain intensity [e.g. measured by visual analog scale (VAS)] (2) swelling (e.g. knee circumference measured using tape), and (3) adverse events (thermal injury such as frostbite and transient nerve palsy).

\section{Search strategy}

The following databases were searched: MEDLINE (via Pubmed, 1990-2014); EMBASE (via Elsevier, 19902014); Cochrane Central Register of Controlled Trials (The Cochrane Library, 1990-2014); CINAHL (19902014), and China National Knowledge Infrastructure (1990-2014). ClinicalTrials.gov was also searched for ongoing and recently completed trials. Studies that were published in English and Chinese were included into this study. The search was complemented by screening the reference lists of retrieved articles. These search strategies were based on the strategy developed for MEDLINE (via Pubmed), combined with the high-pass sensitivity filter developed by the Cochrane Collaboration, to identify RCTs (Higgins and Green 2011). The following search terms were used: "Anterior Cruciate Ligament" OR "Anterior Cruciate Ligament Reconstruction" OR "Arthroplasty, Replacement, Knee" OR "Arthroscopy", AND "Cryotherapy"; and related terms adapted for each database. In addition, in order to search for relevant studies, experts in the field were consulted; and the reference list of all these studies were individually checked for additional studies. Studies published prior to 1990 were excluded due to changes in technology and patient care and populations.

\section{Search outcome}

The initial search identified 593 possible studies. The screening of titles, abstracts and full papers against the inclusion criteria resulted in the selection of ten studies (Fig. 1). Studies with postoperative recovery (including VAS and/or girth measure) records were included in the pooling.

\section{Quality appraisal}

The methodological quality of these trials was assessed through the JADAD scale, a tool for assessing the quality of RCTs, through the evaluation of blinding,

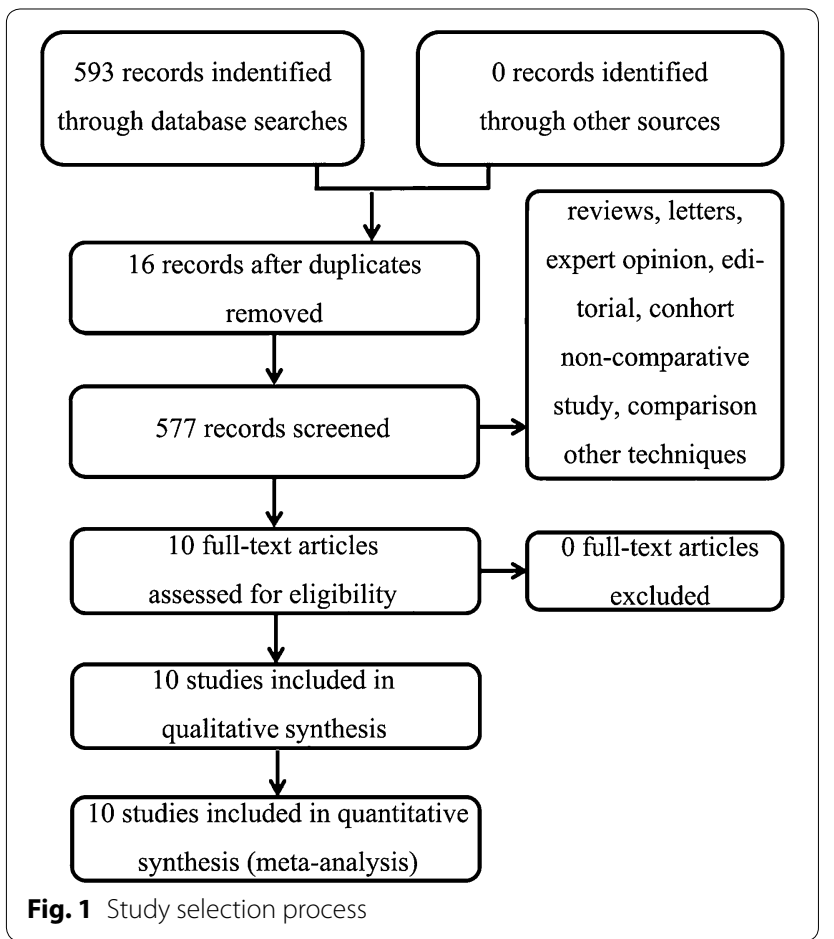

randomization and losses reported (Jadad et al. 1996) (Fig. 2). Additionally, bias in treatment intention, prognosis characteristics, regional differences, amount of losses and follow-up was assessed; but these were not used as exclusion criteria. The full texts of studies that were considered potentially relevant were obtained and read independently by the same two reviewers. Studies that fulfilled the aforementioned selection criteria were included in the meta-analysis. Disagreements between two reviewers were decided by a third reviewer.

\section{Data extraction}

Two independent reviewers (TXL and ZXB) extracted data from all included studies using a standardized extraction form especially created for this meta-analysis. The form contained information of the participants, the methodological aspects of the study, interventions, and measured outcomes. These two individual forms were discussed by the reviewers until a consensus was reached, and these forms were merged into a single extraction form. Persistent disagreements were settled by a third reviewer. When necessary, the authors of these included studies were contacted for further information.

\section{Quantitative data synthesis and analysis}

For the meta-analysis component, raw unstandardized mean differences of VAS and postoperative girth measure (at the joint line level of the operative knee) at 


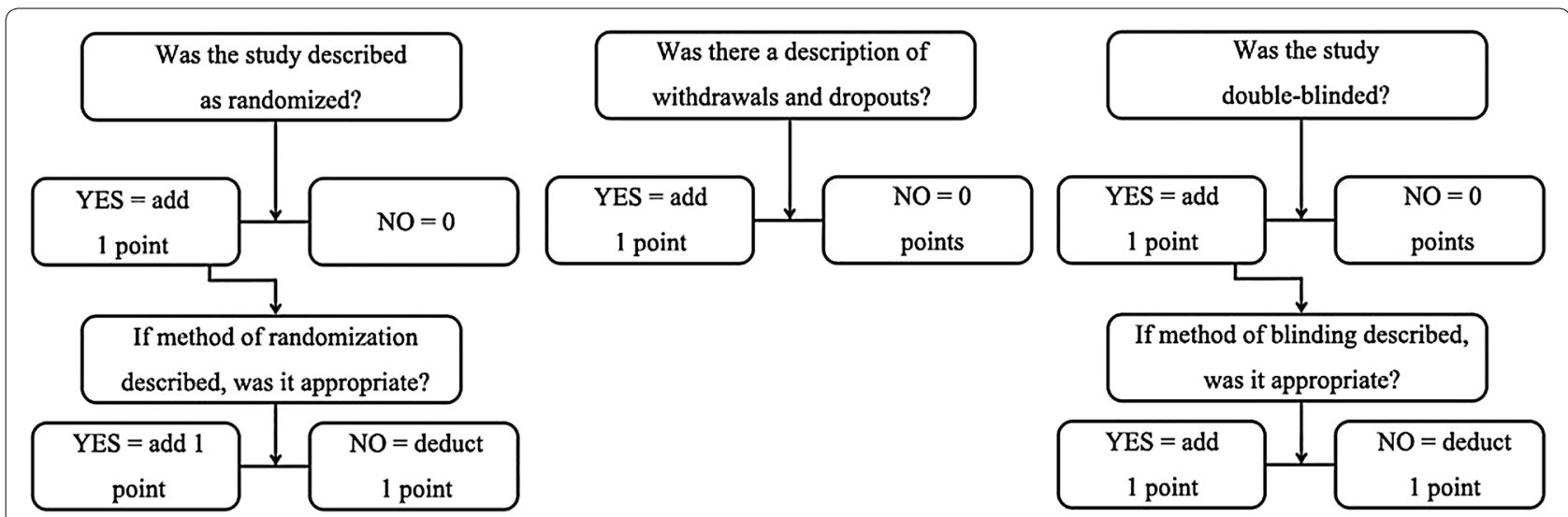

Fig. 2 JADAD scale

postoperative day (POD) $1-3$, as well as at postoperative week (POW) 1 and 2, were extracted from studies that provided these data. This pooling method was selected, because this measure (mean VAS and girth measure) is meaningful for evaluating therapeutic efficacy, well-recognized as valid, and the most common form of measurement across similar studies. VAS scoring with a range of $0-10$ or $0-100$ was used to evaluate subjective pain intensity. Furthermore, VAS scores were used to assess the postoperative pain condition of patients who received $C C$ and $C A$ in all included studies. VAS scores at POD1, POD2, POD3, as well as at POW1 and POW2, were included into the meta-analysis. Girth measure at mid-patella level was commonly performed to evaluate the postoperative swelling condition of patients. For this study, the value of swelling equals the circumference of the operative knee minus the circumference of the healthy knee. Finally, swelling at POD1, POD2 and POD3 were included into the meta-analysis. Due to the likelihood of diversity between the selected trials, settings and populations, a random effects model was used to synthesize these data. The standardized mean difference (SMD) was calculated to allow for two different VAS scales (Lakhan et al. 2015). In addition, heterogeneity was considered to be low when $\mathrm{I}_{2}$ was $\leq 25 \%$, moderate when $\mathrm{I}_{2}$ was $\leq 50 \%$, high when $\mathrm{I}_{2}$ was $\leq 75 \%$, and very high when $\mathrm{I}_{2}$ was $>75 \%$ (Higgins and Green 2011). All calculations were performed via the RevMan 5 software (Review Manager 5.3.5, Cochrane Collaboration). $P$ values were considered statistically significant at $\leq 0.05$.

\section{Results}

\section{Literature search}

A total of 593 potential trials were identified via the first search strategy. Then, 577 reports were excluded during the screening of titles, and six reports were excluded after screening of abstracts. Finally, 583 reports were excluded according to eligibility criteria. No additional studies were obtained after the reference review. After careful full-text evaluation, ten independent RCTs (Schröder and Pässler 1994; Li et al. 2010a, b; Demoulin et al. 2012; Waterman et al. 2012; Tian et al. 2013; Xie et al. 2013, 2014; Xu et al. 2013; Wang et al. 2014) with 522 patients were included in the current meta-analysis.

\section{Study characteristics}

The main characteristics of the included studies are listed in Table 1. The sample size of the included studies ranged from 32 to 140 patients. The methods used for surgery were mostly arthroscopic surgery $(n=7$ trials), ACL reconstruction ( $n=3$ trials) and TKA $(n=2$ trial). Statistically similar baseline characteristics were observed between the $\mathrm{CC}$ and $\mathrm{CA}$ groups, including age and gender. The frequency, number and implementation methods of compression and cryotherapy varied among studies. However, methods used for assessing postoperative pain and swelling were VAS and girth measure, respectively.

\section{Risk of bias assessment}

Based on the JADAD scale, the maximum score among these included studies (Jadad et al. 1996) was 3; because it is not possible to have a double-blind study in this field. Overall methodological quality was moderate (7 trials, JADAD Score $=3$ ), while three trials were of moderate to low quality (JADAD Score $=1$ ). It was not possible to test for publication bias due to the number of trials, in which the outcomes that could be synthesized was too small.

\section{Meta-analysis outcomes VAS scores at POD 1}

Seven included studies included VAS scores at POD1 (Schröder and Pässler 1994; Li et al. 2010a; Tian et al. 


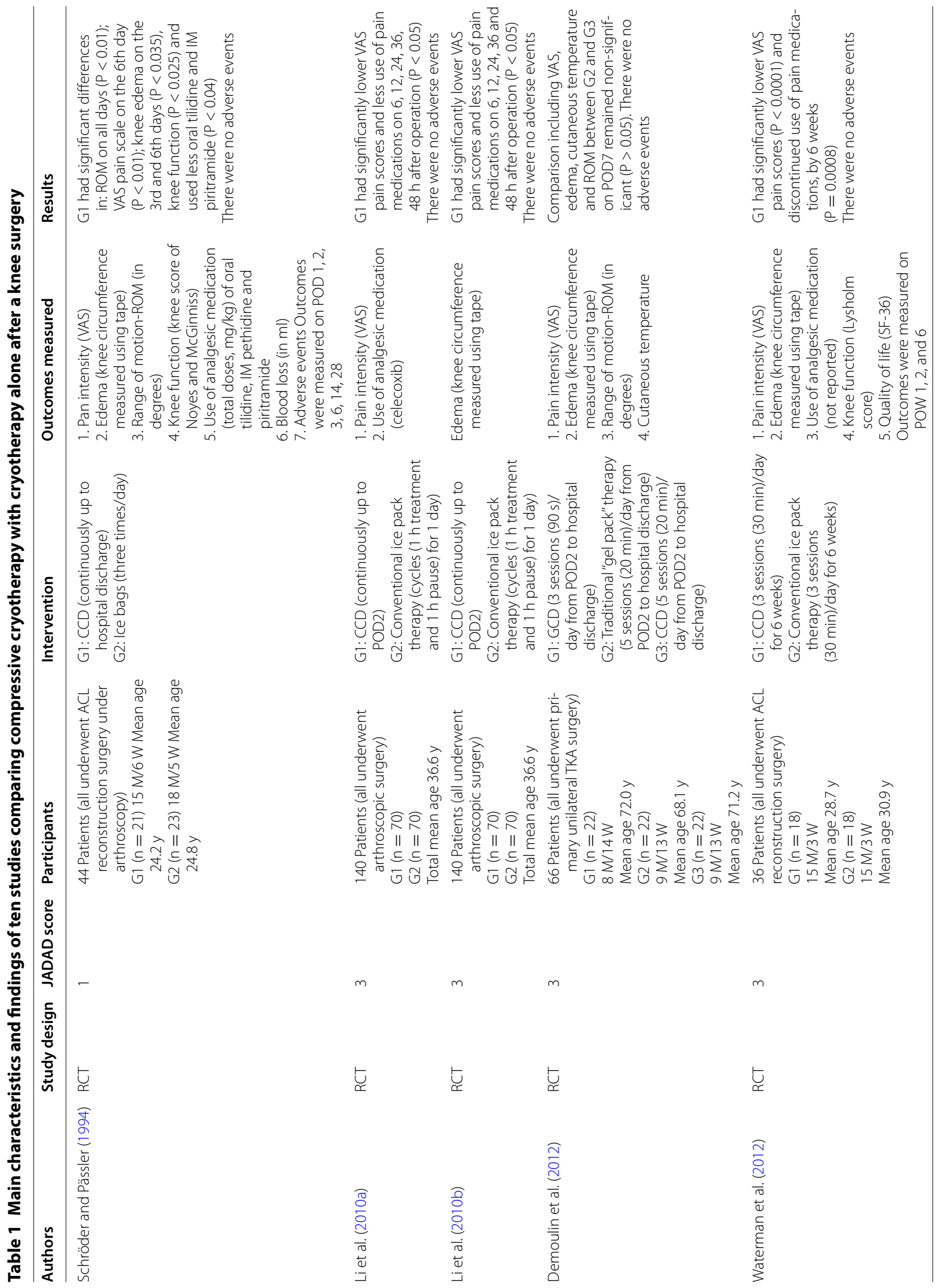




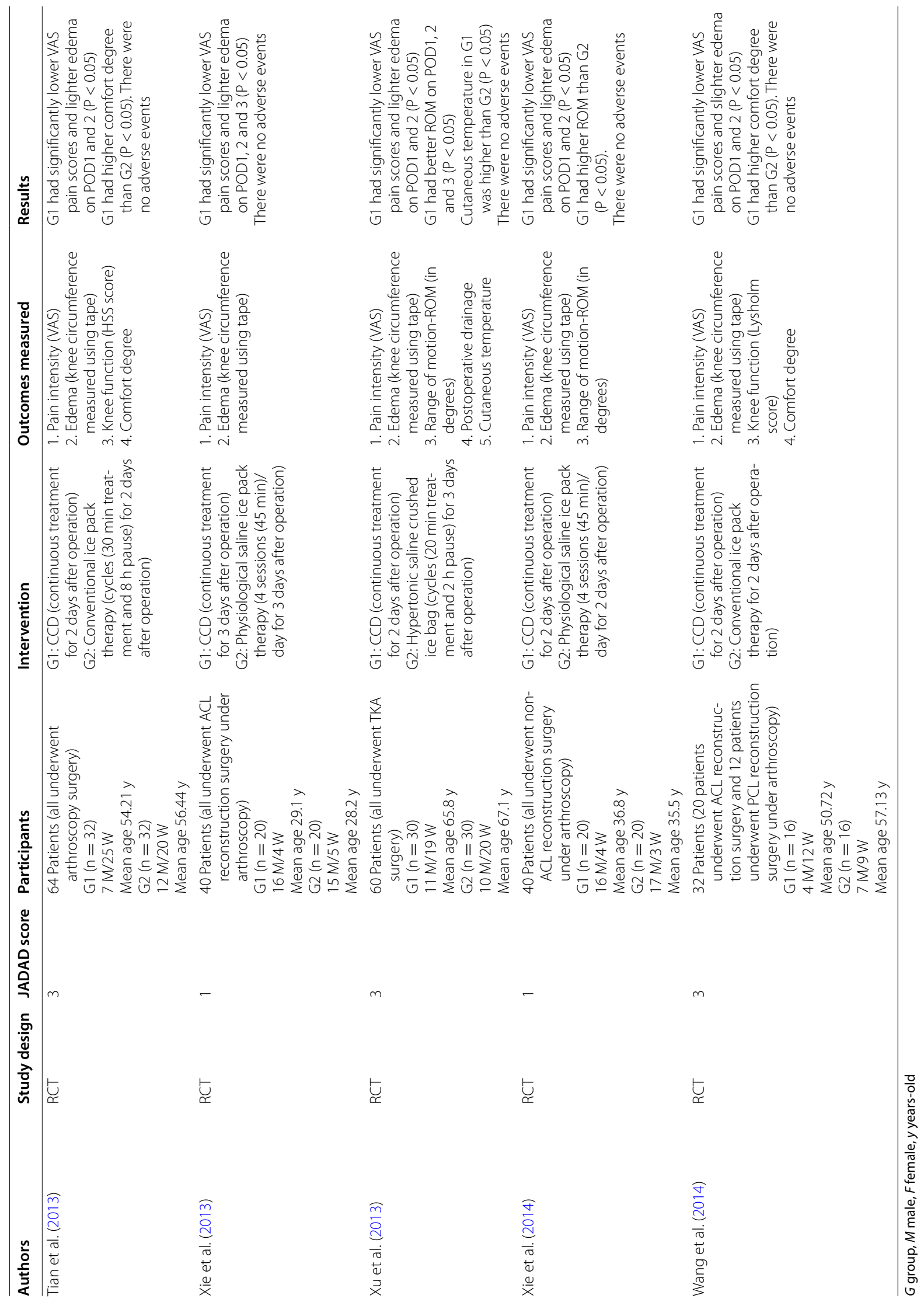


2013; Xie et al. 2013, 2014; Xu et al. 2013; Wang et al. 2014). Although these pooled results have indicated that the remission effect of $\mathrm{CC}$ on postoperative pain was better than that of CA [MD (mean difference) $=-0.94,95 \%$ $\mathrm{CI}-1.63$ to $-0.26, P=0.007$ ], there was significant heterogeneity $\left(\mathrm{Chi}^{2}=60.10 \mathrm{df}=6, I^{2}=90 \%, P<0.00001\right.$; Fig. 3a).

\section{VAS scores at POD2}

Seven studies reported VAS scores at POD2 (Schröder and Pässler 1994; Li et al. 2010a; Tian et al. 2013; Xie et al. 2013, 2014; Xu et al. 2013; Wang et al. 2014), and there was no significant heterogeneity $\left(\mathrm{Chi}^{2}=7.52\right.$, $\mathrm{df}=6, I^{2}=20 \%, P=0.28$; Fig. $3 \mathrm{~b}$ ). Pooled results indicated that the remission effect of $\mathrm{CC}$ was better than that of $\mathrm{CA}$, and there was a statistically significant difference between these two groups ( $\mathrm{MD}=-0.55,95 \% \mathrm{CI}-0.78$ to $-0.32, P<0.00001)$.

\section{VAS scores at POD 3}

Two included studies assessed VAS scores at POD3 (Xie et al. 2013; Xu et al. 2013), and there was no significant heterogeneity $\left(\mathrm{Chi}^{2}=1.33, \mathrm{df}=1, I^{2}=25 \%, P=0.25\right.$; Fig. 3c). Pooled results indicate that the remission effect of CC was better than that of CA, and there was a statistically significant difference between these two groups $(\mathrm{MD}=-0.46,95 \% \mathrm{CI}-0.78$ to $0.38, P=0.03)$.

\section{VAS scores at POW1}

Two included studies assessed VAS scores at POW1 (Demoulin et al. 2012; Waterman et al. 2012), and there was significant heterogeneity $\left(\mathrm{Chi}^{2}=2.38, \mathrm{df}=1\right.$, $I^{2}=58 \%, P=0.12$; Fig. 4a). Pooled results indicated that there was no significant difference in pain remission effect between these two groups ( $\mathrm{MD}=-0.47,95 \% \mathrm{CI}$ -15.72 to $14.77, P=0.95$ ).

\section{VAS scores at POW2}

Two included studies evaluated VAS scores at POW2 (Schröder and Pässler 1994; Waterman et al. 2012), and there was no significant heterogeneity $\left(\mathrm{Chi}^{2}=0.01\right.$, $\mathrm{df}=1, I^{2}=0 \%, P=0.92$; Fig. $4 \mathrm{~b}$ ). Pooled results revealed that there was no significant difference in pain remission effect between these two groups ( $\mathrm{MD}=-1.28,95 \% \mathrm{CI}$ -5.68 to $3.13, P=0.57$ ).

\section{Girth measurement at POD1}

Three studies reported girth measurements at POD1 (Schröder and Pässler 1994; Li et al. 2010b; Xu et al. 2013), and there was no significant heterogeneity $\left(\mathrm{Chi}^{2}=0.44, \mathrm{df}=2, I^{2}=0 \%, P=0.80\right.$; Fig. 5a). Pooled results indicated that swelling in $\mathrm{CC}$ was smaller than in $\mathrm{CA}$, and there was a statistically significant difference between these two groups ( $\mathrm{MD}=-0.19,95 \% \mathrm{CI}-0.23$ to $-0.15, P<0.00001)$.

\section{Girth measurement at POD2}

Three studies reported girth measurements at POD2 (Schröder and Pässler 1994; Li et al. 2010b; Xu et al. 2013), and there was no significant heterogeneity $\left(\mathrm{Chi}^{2}=0.24, \mathrm{df}=2, I^{2}=0 \%, P=0.89\right.$; Fig. 5b). Pooled results indicated that swelling in $\mathrm{CC}$ was smaller than in CA, and there was a statistically significant difference between these two groups ( $\mathrm{MD}=-0.08,95 \% \mathrm{CI}-0.14$ to $-0.02, P=0.01)$.

\section{Girth measurement at POD3}

Two studies reported girth measurement at POD2 (Schröder and Pässler 1994; Xu et al. 2013), and there was no significant heterogeneity $\left(\mathrm{Chi}^{2}=0.00, \mathrm{df}=1\right.$, $I^{2}=0 \%, P=0.99$; Fig. 5c). Pooled results revealed that there was no significant difference in swelling between these two groups ( $\mathrm{MD}=-0.30,95 \% \mathrm{CI}-0.82$ to -0.22 , $P<0.25)$.

\section{Adverse reactions}

No postoperative adverse reactions occurred in all included studies.

\section{Discussion}

Postoperative satisfaction and functional recovery of patients determine the overall efficacy of knee surgery. Consequently, the methods of postoperative management have achieved a rapid advance. Among these methods, supplying a low cost, convenient and satisfactorily effective cryotherapy has been widely recognized and adopted by both clinical and nursing staffs.

Current clinical studies have demonstrated that cryotherapy after knee surgery may significantly bring immediate benefits by decreasing pain and edema during inflammatory response after surgery, reducing muscle spasm, and promoting knee function recovery; thereby accelerating postoperative rehabilitation and the ability of patients to return to routine activities. To the best of our knowledge, cold dressing, cold packs, crushed ice bags and cooling pads have been used as common and traditional methods. With the development of cryotherapy techniques, CCD could simultaneously provide cold and compression to the knee surgery area; and this has become a routine in postoperative cryogenic nursing. Novel devices that generally comprise of specific cuffs, tubes and coolers make the operation simpler, maintain low temperatures for longer periods, and are more appropriate to the operation area. Compared with traditional cryotherapy, CCD brings not only pressurized therapy, but also extra cost. Therefore, better effectiveness for 


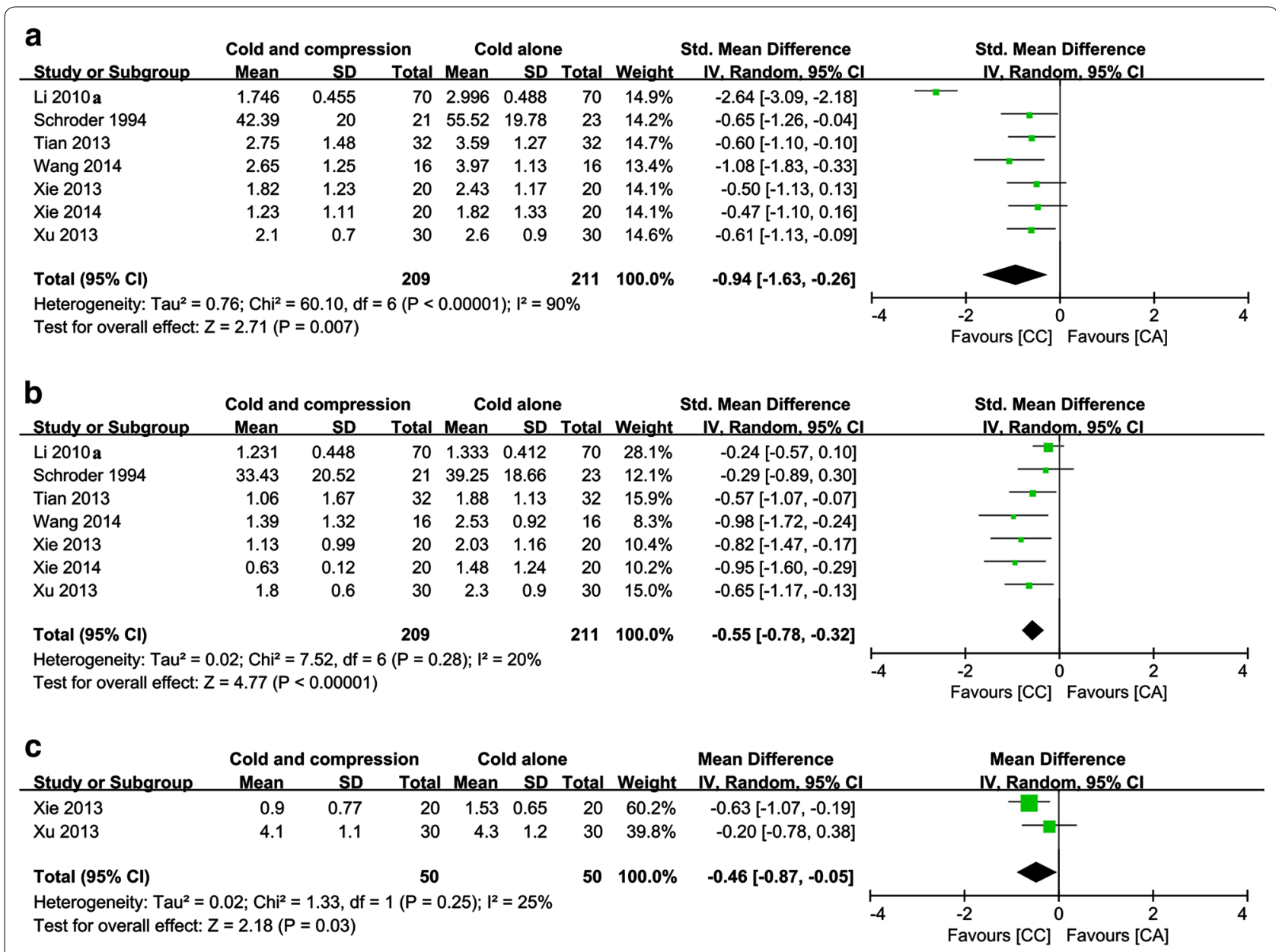

Fig. 3 Pooled data of VAS for compressive cryotherapy versus cryotherapy alone at the early rehabilitation stage: a-c stand for VAS at POD 1,2 and 3 , respectively

postoperative management may become the crucial factor for its application. For CCD, it remains undetermined whether compression or cryotherapy could actually reduce pain and swelling (Martimbianco et al. 2014). However, the limited evidence currently available from randomized trials is insufficient to draw certain conclusions on the comparison of CC and CA, in terms of the effectiveness of pain and swelling.

This meta-analysis included ten RCTs. To summarize, our review of recent English and Chinese literatures revealed that $\mathrm{CC}$ may have better therapeutic effects than CA. Furthermore, our meta-analysis suggests that: (1) patients who underwent $\mathrm{CC}$ had a better analgesic effect than those who underwent CA at POD2 and POD3; (2) CC had a better effect on swelling at POD1 and POD2; (3) it is noteworthy to mention that there were no reported serious adverse events in all included studies. Taken together, these results suggest that the effectiveness of
$\mathrm{CC}$ is better than that of $\mathrm{CA}$ for patients undergoing knee surgery at the early stage of rehabilitation.

No previous meta-analyses have considered the comparison of the effectiveness of $\mathrm{CC}$ and $\mathrm{CA}$ after knee surgery, but several prior analyses were conducted on the effectiveness of CA. The conclusion of the metaanalyses on cryotherapy after knee surgery was common and helpful for clinical practice. Raynor et al. conducted a meta-analysis and revealed that cryotherapy after ACL reconstruction has a statistically significant benefit in postoperative pain control, while no improvement in postoperative range of motion or drainage was found (Raynor et al. 2005). By analyzing ten trials, the study of Martimbianco et al. was found to reveal that the use of CCD produced a significant reduction in pain scores at POD2 after arthroscopic ACL surgery compared to that without cryotherapy (Martimbianco et al. 2014). In brief, cryotherapy is effective for pain relief and swelling-control 


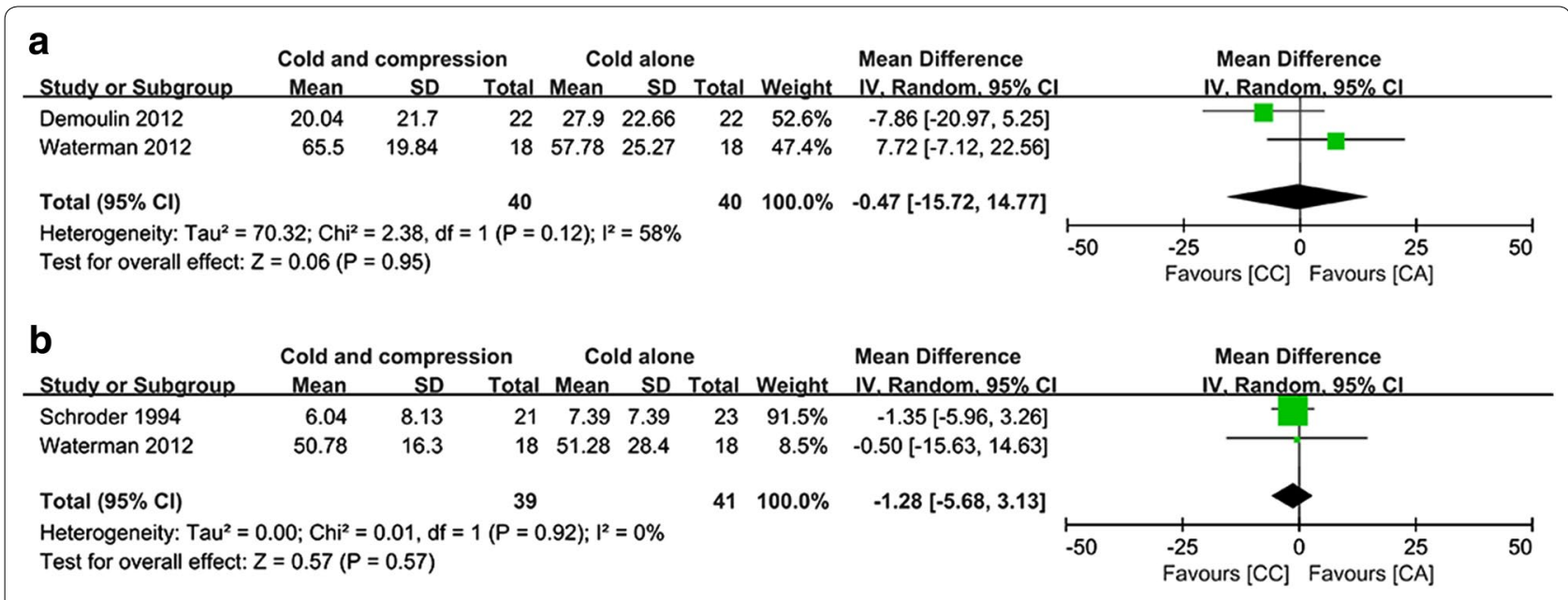

Fig. 4 Pooled data of VAS for compressive cryotherapy versus cryotherapy alone at the chronic rehabilitation stage: a, b stand for VAS at POW 1 and 2 , respectively

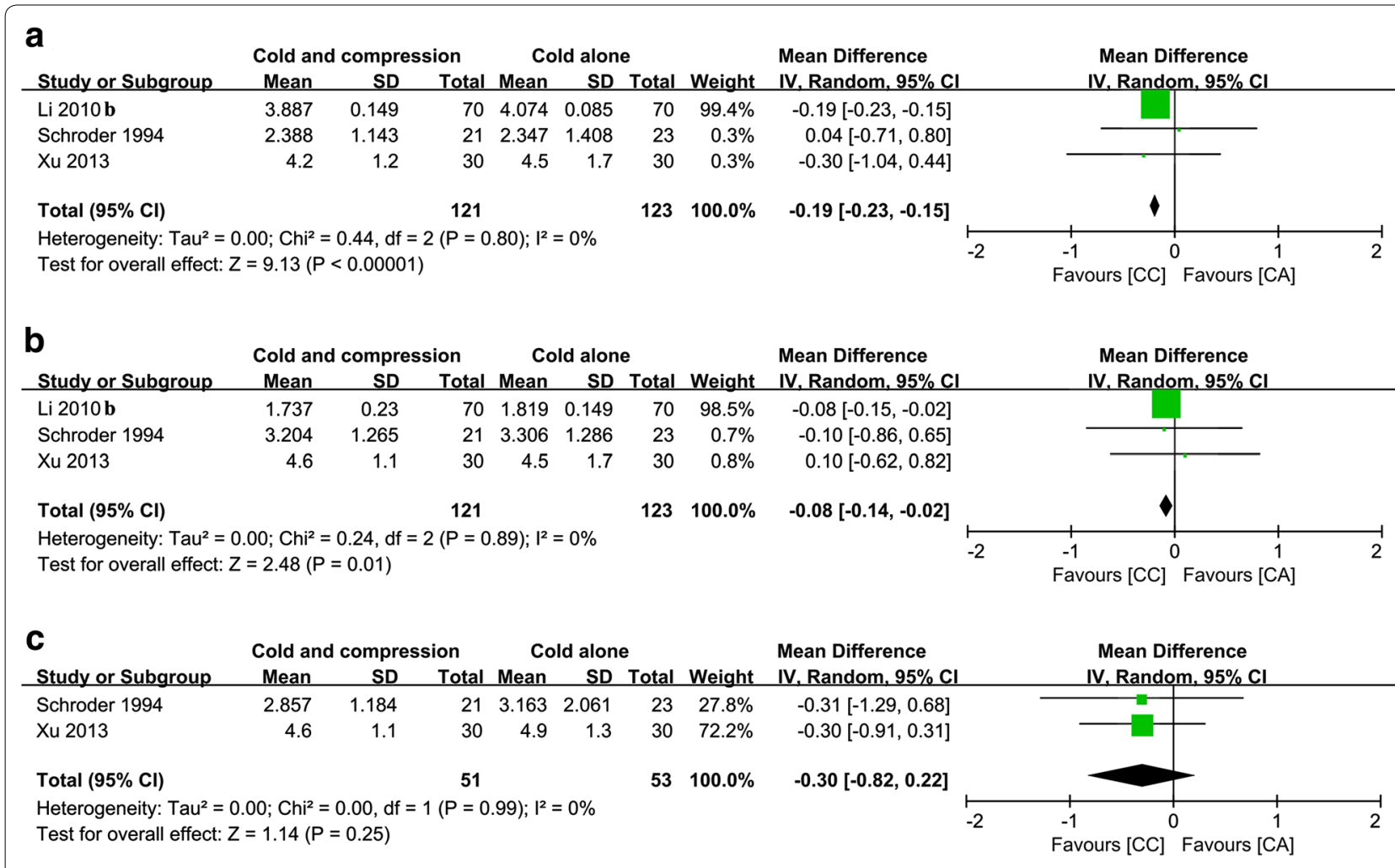

Fig. 5 Pooled data of girth measure for compressive cryotherapy versus cryotherapy alone at the early rehabilitation stage: a-c stand for swelling at POD 1, 2 and 3, respectively

in patients undergoing knee surgery, especially at the early postoperative stage. In terms of our analysis results, CC was more effective than CA for pain remission at POD2 and POD3. Additionally, we found that $\mathrm{CC}$ had more advantages for decreasing swelling at POD1 and POD2. Based on cryotherapy, compression played an important role in pain relief and swelling-control. Theoretically, the persistence of vasoconstriction may be the main cause 
for coping with soft tissue injuries, through modulating swelling, pain, inflammation, metabolism, muscle spasm, and bleeding (Bleakley et al. 2004; Schaser et al. 2007). In practice, the dressing of low temperatures on the skin surface effectively enhances the healing of soft tissue injuries (Mejia et al. 2015). Therefore, the application of lower temperatures can reduce the need for pain medications and promote recovery (Trobec et al. 2008). In addition, Adie et al. consider that a low-temperature state might be able to reduce swelling by decreasing postoperative blood loss (Adie et al. 2012). However, the related molecular mechanism remains unclear. According to the conclusion of different meta-analyses, CA has been regarded as an effective pain relief and swelling-control nursing management for postoperative patients (Ni et al. 2015; Adie et al. 2010; Martimbianco et al. 2014). Furthermore, a systematic review on cryotherapy for acute soft tissue injury revealed the small but statistically significant effect of CC compared to CA (Bleakley et al. 2004). In concordance with other researchers' studies, our outcome indicated that compression combined with cryotherapy effectively enhanced the curative effect. Due to restraints in the quantity of included trials and patients, no adverse reactions were reported in any of the included trials. However, adverse reactions of cryotherapy such as frostbite, cutaneous necrosis and neuropathy should not be ignored (Khoshnevis et al. 2015). Interestingly, soft tissue damage due to compression could be reduced by lowering the temperature, although compression, and has a potential impact on skin perfusion. This result may be connected with proinflammatory cytokine accumulation (Lee et al. 2014). To date, only one patient in the ice pack group developed transient peroneal nerve palsy, because cryotherapy time lasted for nearly $40 \mathrm{~min}$ (Cohn et al. 1989). Since $30 \mathrm{~min}$ has been generally adopted for cryotherapy treatment, no other adverse reactions were found.

Although the level of evidence was relatively low, this evidence still provided partial answers to the core questions raised in our study. In most included trials, the evaluation of outcomes was limited to a short observation period, which was between POD1 and POD3. Few English studies have evaluated the results of postoperative intervention. Therefore, it is necessary to perform further analyses that would include more sufficient longterm outcomes.

All studies that were included in this review had high risk of bias, recruited a small number of patients, and provided sparse data on most of our pre-established outcomes of interest; thereby precluding the pooling of their results into these meta-analyses. Furthermore, these studies were heterogenous in several aspects: these trials applied different forms of knee surgery (TKA, knee arthroplasty, ACL reconstruction and arthroscopic therapy), CA (ice bag and ice pack), CC (equal to CCD including Cryo Cuff system, Game ready coolsystems and Ever-cryo system), different frequencies and durations during sessions, and different follow-up periods. Inevitably, ice bag, cold pack and CCD also differ in handling, effect and efficiency. The main methodological limitations of these included studies were the lack of description of allocation concealment, difficulties in the blinding of participants, and outcome assessors; which were due to the nature of the intervention. This may in part be explained by the fact that old English and Chinese trials did not apply the standard recommendations for reporting clinical trials. Further studies with more consistent cryotherapy measurements and more standard data records would help to more accurately confirm this conclusion.

\section{Conclusion}

$\mathrm{CC}$ and $\mathrm{CA}$ are both safe management methods for patients undergoing knee surgery. There is a moderate quality of evidence that $\mathrm{CC}$ is more effective in reducing pain at POD2 and POD3, coping with swelling at POD1 and POD2 after knee surgery. For patients who can afford CCD, we thought that these could obtain more benefits by applying CC at POD 1-3 after knee surgery. After the early stage, patients can have a choice (CC or CCD) for the remaining rehabilitation stages. The limited evidence currently available is insufficient to draw definitive conclusions on the effectiveness of this intervention for other outcomes such as the consumption of postoperative analgesic medications, knee range of motion, blood loss, hospital stay duration, quality of life measures and patient satisfaction. Moreover, welldesigned, high quality randomized trials are needed to answer unsolved questions related to this comparison, as well as to supply more evidence-based conclusions and suggestions.

\section{Relevance to clinical practice}

This review has provided an important contribution in selecting the optimum method for cryotherapy following knee surgery, by conducting a comparative evaluation between compressive cryotherapy and cryotherapy alone. More research is needed in this area to gain sufficient knowledge on other outcomes such as the consumption of postoperative analgesic medications, knee range of motion, blood loss, hospital stay duration, quality of life measures and patient satisfaction; since the current evidence obtained for this is weak. Additionally, more well-designed, high quality randomized trials are also expected. 


\section{Abbreviations}

TKA: total knee arthroplasty; ACL: anterior cruciate ligament; CCD: cold compression devices; CC: compressive cryotherapy; CA: cryotherapy alone; VAS: visual analog scale; POD: postoperative day; POW: postoperative week; MD: mean difference; SMD: standardized mean difference.

\section{Authors' contributions}

SMZ and SR were responsible for the conception and design of the study, contributed to the collection, analysis and interpretation of data, and responsible for drafting, revising the manuscript. STY, SXH and DW assisted in the revision of the manuscript. ZXB and TXL extracted data from all included studies. All authors read and approved the final manuscript.

\section{Author details}

${ }^{1}$ Department of Orthopaedics, The First Affiliated Hospital of Dalian Medical University, 222 Zhong Shan Road, Dalian 116011, Liaoning, People's Republic of China. ${ }^{2}$ Department of Orthopaedics, The Third Affiliated Hospital of Dalian Medical University, 378 Shi Ji West Road, Jinpu New Area 116200, Liaoning, People's Republic of China. ${ }^{3}$ Department of Nursing, The First Affiliated Hospital of Dalian Medical University, 222 Zhong Shan Road, Dalian 116011, Liaoning, People's Republic of China. ${ }^{4}$ Department of Hepatobiliary Surgery, The First Affiliated Hospital of Dalian Medical University, 222 Zhong Shan Road, Dalian 116011, Liaoning, People's Republic of China. ${ }^{5}$ Operation Room, The First Affiliated Hospital of Dalian Medical University, 222 Zhong Shan Road, Dalian 116011, Liaoning, People's Republic of China.

\section{Acknowledgements}

None.

\section{Competing interests}

The authors declare that they have no competing interests.

Received: 26 February 2016 Accepted: 23 June 2016

Published online: 13 July 2016

\section{References}

Adie S, Naylor JM, Harris IA (2010) Cryotherapy after total knee arthroplasty a systematic review and meta-analysis of randomized controlled trials. J Arthroplasty 25:709-715

Adie S, Kwan A, Naylor JM, Harris IA, Mittal R (2012) Cryotherapy following total knee replacement. Cochrane Database Syst Rev 12:7911

Andersen L $\varnothing$, Husted H, Otte KS, Kristensen BB, Kehlet H (2008) A compression bandage improves local infiltration analgesia in total knee arthroplasty. Acta Orthop 79:806-811

Bleakley C, McDonough S, MacAuley D (2004) The use of ice in the treatment of acute soft-tissue injury: a systematic review of randomized controlled trials. Am J Sports Med 32:251-261

Charalambides C, Beer M, Melhuish J, Williams RJ, Cobb AG (2005) Bandaging technique after knee replacement. Acta Orthop 76:89-94

Cheung A, Lykostratis H, Holloway I (2014) Compression bandaging improves mobility following total knee replacement in an enhanced recovery setting. J Perioper Pract 24:84-86

Cohn BT, Draeger RI, Jackson DW (1989) The effects of cold therapy in the postoperative management of pain in patients undergoing anterior cruciate ligament reconstruction. Am J Sports Med 17:344-349

Demoulin C, Brouwers M, Darot S, Gillet P, Crielaard JM, Vanderthommen M (2012) Comparison of gaseous cryotherapy with more traditional forms of cryotherapy following total knee arthroplasty. Ann Phys Rehabil Med 55:229-240

Higgins JPT, Green S (2011) Cochrane handbook for systematic reviews of interventions. The Cochrane collaboration. Version 5.0.2 www.cochranehandbook.org

Jadad AR, Moore RA, Caroll D, Jenkinson C, Reynolds DJM, Gavaghan DJ, McQuay HJ (1996) Assessing the quality of reports of randomized clinical trials: is blinding necessary? Control Clin Trials 17:1-12

Khoshnevis S, Craik NK, Diller KR (2015) Cold-induced vasoconstriction may persist long after cooling ends: an evaluation of multiple cryotherapy units. Knee Surg Sports Traumatol Arthrosc 23:2475-2483
Kraeutler MJ, Reynolds KA, Long C, McCarty EC (2015) Compressive cryotherapy versus ice-a prospective, randomized study on postoperative pain in patients undergoing arthroscopic rotator cuff repair or subacromial decompression. J Shoulder Elbow Surg 24:854-859

Lakhan SE, Ford CT, Tepper D (2015) Zingiberaceae extracts for pain: a systematic review and meta-analysis. Nutr J 14:50

Lee B, Benyajati S, Woods JA, Jan YK (2014) Effect of local cooling on proinflammatory cytokines and blood flow of the skin under surface pressure in rats: feasibility study. J Tissue Viability 23:69-77

Lessard LA, Scudds RA, Amendola A, Vaz MD (1997) The efficacy of cryotherapy following arthroscopic knee surgery. J Orthop Sports Phys Ther 26:14-22

Li LL, Ning N, Hou XL, Zhu H, Chen JL, Chen ZL, Liao DB, Yang H (2010a) Clinical research on the Cryo/cuff system in controlling pain of postoperative knee arthroscopy patients. Mod Prev Med 37:2587-2589 (article in Chinese)

Li LL, Ning N, Hou XL, Zhu H, Chen JL, Chen ZL (2010b) Cryo/Cuff system controls swelling following postoperative knee arthroscopy patients. J Clin Rehabil Tissue Eng Res 14:7286-7389 (article in Chinese)

Markert SE (2011) The use of cryotherapy after a total knee replacement: a literature review. Orthop Nurs 30:29-36

Martimbianco AL, Gomes da Silva BN, de Carvalho AP, Silva V, Torloni MR, Peccin MS (2014) Effectiveness and safety of cryotherapy after arthroscopic anterior cruciate ligament reconstruction. A systematic review of the literature. Phys Ther Sport 15:261-268

Mejia N, Dedow K, Nguy L, Sullivan P, Khoshnevis S, Diller KR (2015) An On-Site Thermoelectric Cooling Device for Cryotherapy and Control of Skin Blood Flow. J Med Device 9:445021-445026

Meyer-Marcotty M, Jungling O, Vaske B, Vogt PM, Knobloch K (2011) Standardized combined cryotherapy and compression using Cryo/Cuff after wrist arthroscopy. Knee Surg Sports Traumatol Arthrosc 19:314-319

Munk S, Jensen NJ, Andersen I, Kehlet H, Hansen TB (2013) Effect of compression therapy on knee swelling and pain after total knee arthroplasty. Knee Surg Sports Traumatol Arthrosc 21:388-392

Nadler SF, Weingand K, Kruse RJ (2004) Cryotherapy and thermotherapy for the pain practitioner. Pain Physician 7:395-399

Ni SH, Jiang WT, Guo L, Jin YH, Jiang TL, Zhao Y, Zhao J (2015) Cryotherapy on postoperative rehabilitation of joint arthroplasty. Knee Surg Sports Traumatol Arthrosc 23:3354-3361

Pinsornsak P, Chumchuen S (2013) Can a modified Robert Jones bandage after knee arthroplasty reduce blood loss? A prospective randomized controlled trial. Clin Orthop Relat Res 471:1677-1681

Raynor MC, Pietrobon R, Guller U, Higgins LD (2005) Cryotherapy after ACL reconstruction: a meta-analysis. J Knee Surg 18:123-129

Schaser KD, Disch AC, Stover JF, Lauffer A, Bail HJ, Mittlmeier T (2007) Prolonged superficial local cryotherapy attenuates microcirculatory impairment, regional inflammation, and muscle necrosis after closed soft tissue injury in rats. Am J Sports Med 35:93-102

Schröder D, Pässler HH (1994) Combination of cold and compression after knee surgery: A prospective randomized study. Knee Surg Sports Traumatol Arthrosc 2:158-165

Smith J, Stevens J, Taylor M, Tibbey J (2002) A randomized, controlled trial comparing compression bandaging and cold therapy in postoperative total knee replacement surgery. Orthop Nurs 21:61-66

Tian RX, Hao YF, Guo H (2013) Comparison of Cryo/Cuff system with ice com press in postoperative care of knee arthroscopy patients. J Nurs (China) 20:10-13 (article in Chinese)

Trobec R, Sterk M, AlMawed S, Veselko M (2008) Computer simulation of topical knee cooling. Comput Biol Med 38:1076-1083

van Grinsven S, van Cingel RE, Holla CJ, van Loon CJ (2010) Evidence-based rehabilitation following anterior cruciate ligament reconstruction. Knee Surg Sports Traumatol Arthrosc 18:1128-1144

Wang FF, Li Q, Wei YL (2014) The effectiveness evaluation of cycling compressive cryotherapy system after arthroscopic cruciate ligament reconstruction surgery. China Health Care Nutr 24:3722-3723 (article in Chinese)

Warren TA, McCarty EC, Richardson AL, Michener T, Spindler KP (2004) Intraarticular knee temperature changes: ice versus cryotherapy device. Am J Sports Med 32:441-445

Waterman B, Walker JJ, Swaims C, Shortt M, Todd MS, Machen SM, Owens BD (2012) The Efficacy of Combined Cryotherapy and Compression Compared with Cryotherapy Alone Following Anterior Cruciate Ligament Reconstruction. J Knee Surg 25:155-160 
Xie Q, Huang HY, Duan JF, Chen YR, Zheng XF, Zuo D, Wang YQ, Zhang Y, Xia H (2013) The effect of continuous compression cryotherapy for postoperative pain and swelling after anterior cruciate ligament reconstruction. China J Rehabil 28:190-191 (article in Chinese)

Xie Q, Liu H, Huang HY, Wang YQ, Li PY, Zheng XF, Yin QS (2014) The early effect of continuous compression cryotherapy for postoperative patients undergoing knee arthroscopic operation. Guangdong Med J 35:3-5 (article in Chinese)
Xu XH, Li XF, Xiong YF (2013) Comparison of Cryo/cuff Systems and intermittent cold compress with hypertonic saline following total knee arthroplasty. China J Tissue Eng Res 17:8325-8330 (article in Chinese)

Yabroudi MA, Irrgang JJ (2013) Rehabilitation and return to play after anatomic anterior cruciate ligament reconstruction. Clin Sports Med 32:165-175

\section{Submit your manuscript to a SpringerOpen ${ }^{\odot}$ journal and benefit from:}

- Convenient online submission

- Rigorous peer review

- Immediate publication on acceptance

- Open access: articles freely available online

- High visibility within the field

- Retaining the copyright to your article 